MZ-TH/05-05

\title{
Scalar diagrammatic rules for Born amplitudes in QCD
}

\author{
Christian Schwinn and Stefan Weinzierl \\ Institut für Physik, Universität Mainz, \\ D - 55099 Mainz, Germany
}

\begin{abstract}
We show that all Born amplitudes in QCD can be calculated from scalar propagators and a set of three- and four-valent vertices. In particular, our approach includes amplitudes with any number of quark pairs. The quarks may be massless or massive. The proof of the formalism is given entirely within quantum field theory.
\end{abstract}




\section{Introduction}

The computation of QCD amplitudes is vital to extract information from ongoing and future collider experiments. Even at Born level, the calculation of amplitudes with a large number of external legs is far from trivial. Efficient methods employed up to now start from a decomposition of QCD Born amplitudes into gauge-independent colour structures [1-5]. The expression multiplying a specific colour structure is usually called a partial amplitude. The partial amplitudes can be calculated by using the spinor helicity formalism [6-10] and by using recursive techniques $[11,12]$.

It has been known for a long time, that helicity amplitudes for specific helicity combinations have a remarkably simple analytic formula or vanish altogether $[11,13]$. In particular, the pure gluon amplitude vanishes if all gluons have the same helicity, or if all gluons except one have the same helicity. The first non-vanishing amplitude is obtained if $n-2$ gluon have one type of helicity, and 2 gluons the other type. The $n$-gluon amplitude with $n-2$ gluons of positive helicity and 2 gluons of negative helicity is called a maximal-helicity violating amplitude (MHV amplitude). Similar, the $n$-gluon amplitude with 2 gluons of positive helicity and $n-2$ gluons of negative helicity is often referred to as $\overline{\mathrm{MHV}}$-amplitude or "googly" amplitude. For these amplitudes compact analytical formulae are known.

Recently, Cachazo, Svrček and Witten [14] proposed that the gluon amplitude with an arbitrary helicity configuration can be calculated from diagrams with scalar propagators and new vertices, which are MHV-amplitudes continued off-shell. This conjecture was inspired by insight gained from the transformation of the amplitude to twistor space [15]. In the latter publication, Witten conjectured that the $n$-gluon amplitude with $l$-loops is non-zero only if all points lie in twistor space on an algebraic curve of degree $d$. The degree $d$ of this curve is given by the number of negative helicity gluons plus the number of loops minus one.

These two conjectures triggered significant interest in the community and have been checked by explicit calculations of Born amplitudes [16-21]. As the conjecture on twistor space makes a statement on loop amplitudes, even one-loop amplitudes have been studied extensively [22-41]. Within this context it is worth mentioning some earlier work on twistor methods used to simplify the calculation of Feynman diagrams [42-45].

Of practical interest for the calculation of Born amplitudes in QCD are recursive methods. These have been considered for the pure gluon case in [46-48]. Furthermore, QCD amplitudes may involve in addition to gluons also quark pairs. It has been known for a long time, that Born amplitudes with massless quarks may be related to pure gluon amplitudes through supersymmetric relations $[49,50]$. This is related to the fact that in partial amplitudes, where all colour information has been stripped off, nothing distinguishes a (massless) quark from a gluino. QCD amplitudes with massless quarks have been studied within the context of the MHV-approach in [51-58]. Furthermore, the inclusion of additional non-QCD-type particles, like vector bosons or the Higgs boson have been studied as well [59-61].

In this paper we would like to extend the studies towards massive quarks. We consider Born amplitudes in QCD with any number of quark pairs. The quarks may be massless or massive. This allows for the application of our results towards processes involving the top quark. With massive quarks we can no longer rely on super-symmetric relations and we develop therefore an 
alternative diagrammatic approach. We show that all Born amplitudes in QCD can be calculated from scalar propagators and a set of three- and four-valent vertices. We call these vertices "primitive vertices" to distinguish them from the vertices of the standard Feynman rules on the one hand, and from the MHV-vertices on the other hand. A helicity label $\{+,-\}$ is attached to each leg of a primitive vertex and propagators connect "+"-labels with "-"-labels. One can define the degree of a vertex as the number of "-"-labels minus one. We show that only primitive vertices of degree zero and one occur. The proof of the formalism is given entirely within quantum field theory.

The benefits of our approach are two-fold: First, we are able to treat all QCD partons on an equal footing, independently if they are massless or not. This allows the application of our approach towards problems involving massive quarks. As all propagators are scalars no contraction of Lorentz- or spinor-indices is present any more. This makes our method well suited for a fast implementation on a computer.

Secondly, we see the structure of the Cachazo - Svrček - Witten prescription emerge from our diagrammatic approach: As the degree of an amplitude is exactly the sum of the degrees of the primitive vertices, a MHV amplitude is of degree one and contains exactly one primitive vertex of degree one, which is dressed up in all possible ways with degree zero vertices. Similar, a pure gluon amplitude with three gluons of negative helicities is of degree two and contains two vertices of degree one, which again are combined in all possible ways with degree zero vertices. In general, the pure gluon amplitude with $n_{-}$gluons of negative helicity contains $\left(n_{-}-1\right)$ primitive vertices of degree one, which are supplemented in all possible ways with degree zero vertices.

This paper is organised as follows: In the next section we introduce our notation and review basic facts on QCD amplitudes. In section 3 we show that Born amplitudes in QCD can be calculated from scalar propagators and a set of vertices. In section 4 we discuss the combinatorial aspects of our approach. Section 5 contains our conclusions. In appendix A we list useful information on spinors. We summarise the scalar diagrammatic rules in appendix B.

\section{Notation and review of basic facts on QCD amplitudes}

In this paper we use the normalisation

$$
\operatorname{Tr} T^{a} T^{b}=\frac{1}{2} \delta^{a b}
$$

for the colour matrices. Amplitudes in QCD may be decomposed into group-theoretical factors (carrying the colour structures) multiplied by kinematic functions called partial amplitudes [1-5]. These partial amplitudes do not contain any colour information and are gauge-invariant objects.

The colour decomposition is obtained by replacing the structure constants $f^{a b c}$ by

$$
i^{a b c}=2\left[\operatorname{Tr}\left(T^{a} T^{b} T^{c}\right)-\operatorname{Tr}\left(T^{b} T^{a} T^{c}\right)\right]
$$


which follows from $\left[T^{a}, T^{b}\right]=i f^{a b c} T^{c}$. The resulting traces and strings of colour matrices can be further simplified with the help of the Fierz identity :

$$
T_{i j}^{a} T_{k l}^{a}=\frac{1}{2}\left(\delta_{i l} \delta_{j k}-\frac{1}{N} \delta_{i j} \delta_{k l}\right) .
$$

In the pure gluonic case tree level amplitudes with $n$ external gluons may be written in the form

$$
\mathcal{A}_{n}(1,2, \ldots, n)=g^{n-2} \sum_{\sigma \in S_{n} / Z_{n}} 2 \operatorname{Tr}\left(T^{a_{\sigma(1)}} \ldots T^{a_{\sigma(n)}}\right) A_{n}(\sigma(1), \ldots, \sigma(n)),
$$

where the sum is over all non-cyclic permutations of the external gluon legs. The quantities $A_{n}(\sigma(1), \ldots, \sigma(n))$, called the partial amplitudes, contain the kinematic information. They are colour-ordered, e.g. only diagrams with a particular cyclic ordering of the gluons contribute.

The colour decomposition for a tree amplitude with a pair of quarks is

$$
\mathcal{A}_{n+2}(q, 1,2, \ldots, n, \bar{q})=g^{n} \sum_{S_{n}}\left(T^{a_{\sigma(1)}} \ldots T^{a_{\sigma(n)}}\right)_{i_{q} j_{\bar{q}}} A_{n+2}(q, \sigma(1), \sigma(2), \ldots, \sigma(n), \bar{q}) .
$$

where the sum is over all permutations of the gluon legs. Similar decompositions may be obtained for amplitudes with more than one pair of quarks.

The calculation of partial amplitudes in QCD is the main subject of this paper. In principle, they can be calculated in a helicity basis from all Feynman diagrams with a fixed cyclic ordering. The Feynman rules for colour-ordered partial amplitudes read:

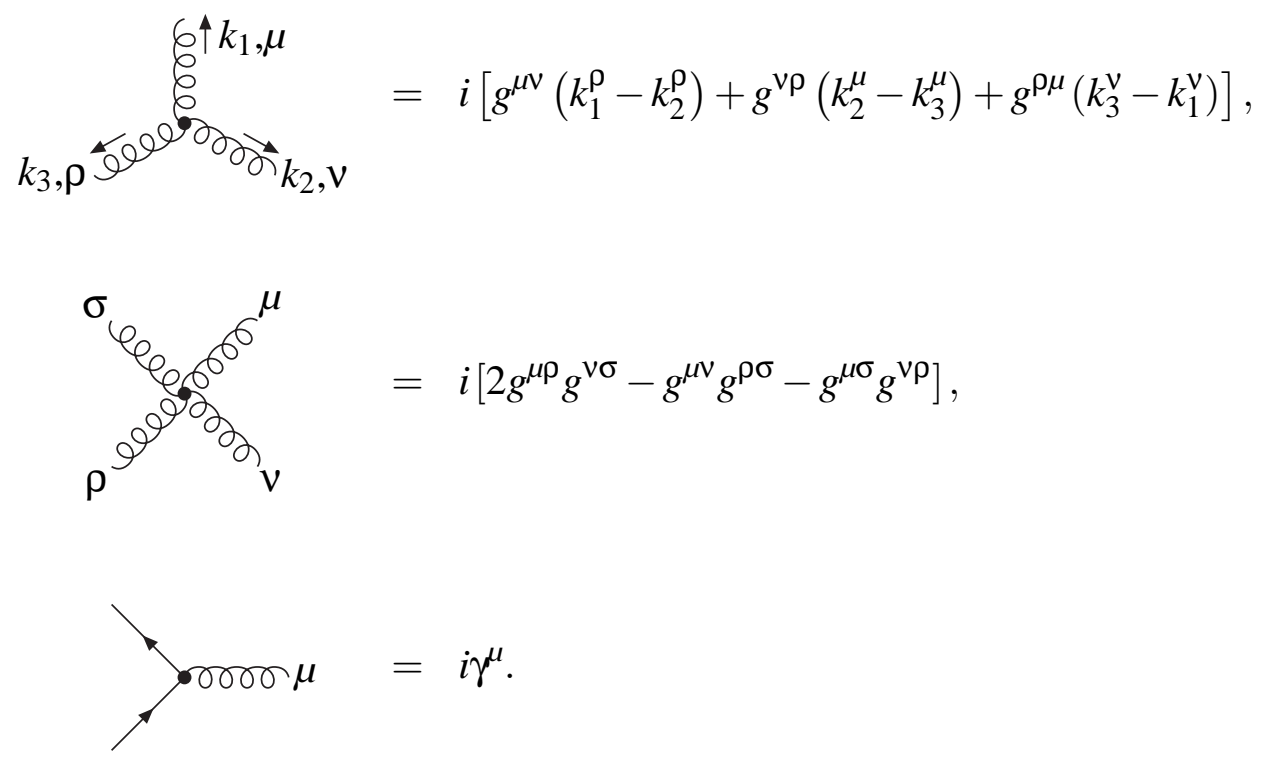

For the calculation of helicity amplitudes [6-10] one chooses for the spinors corresponding to external massless quarks two-component Weyl spinors $\langle p \pm|$ and $|p \pm\rangle$. We use here the bra-ketnotation. The relation with the notation using dotted and undotted indices is as follows:

$$
|p+\rangle=p_{B}, \quad\langle p+|=p_{\dot{A}},
$$




$$
|p-\rangle=p^{\dot{B}}, \quad\langle p-|=p^{A}
$$

For the polarisation vectors of the external gluons one uses

$$
\varepsilon_{\mu}^{+}(k, q)=\frac{\left\langle q-\left|\gamma_{\mu}\right| k-\right\rangle}{\sqrt{2}\langle q-\mid k+\rangle}, \quad \varepsilon_{\mu}^{-}(k, q)=\frac{\left\langle q+\left|\gamma_{\mu}\right| k+\right\rangle}{\sqrt{2}\langle k+\mid q-\rangle},
$$

where $k$ is the momentum of the gluon and $q$ is an arbitrary light-like reference momentum. It is possible to extend the helicity formalism to massive spinors [62-66]. We have collected all necessary details on spinors in appendix A. Our conventions are such that we always take all gluon momenta outgoing and the momenta of quarks to flow in the direction of the fermion lines.

\section{Scalar propagators and primitive vertices}

In this section we show that QCD amplitudes can be calculated from scalar propagators and a set of three- and four-valent vertices. We start by recalling the off-shell continuation of spinors in paragraph 3.1. We then treat first the pure gluonic case in paragraph 3.2. The complete discussion carries over to quarks, which are dealt with in paragraph 3.3.

\subsection{Off-shell continuation}

Let $q$ be a null-vector, which will be kept fixed throughout the discussion. Using $q$, any massive vector $k$ can be written as a sum of two null-vectors $k^{b}$ and $q$ [20]:

$$
k=k^{b}+\frac{k^{2}}{2 k q} q .
$$

Obviously, if $k^{2}=0$, we have $k=k^{b}$. Note further that $2 k q=2 k^{b} q$. Using eq. (10) we may associate to any four-vector $k$ a massless four-vector $k^{b}$. Using the projection onto $k^{b}$ we define the off-shell continuation of Weyl spinors as

$$
\begin{aligned}
|k \pm\rangle & \rightarrow\left|k^{b} \pm\right\rangle, \\
\langle k \pm| & \rightarrow\left\langle k^{b} \pm\right| .
\end{aligned}
$$

We are going to use the following abbreviations:

$$
\begin{aligned}
& \langle i j\rangle=\left\langle k_{i}^{b}-\mid k_{j}^{b}+\right\rangle, \quad[i j]=\left\langle k_{i}^{b}+\mid k_{j}^{b}-\right\rangle, \\
& \langle i-|j \pm k| l-\rangle=\left\langle k_{i}^{b}-\left|k_{j}^{b} \pm k_{k}^{b}\right| k_{l}^{b}-\right\rangle .
\end{aligned}
$$

Let us define an "off-shell amplitude"

$$
O_{n}\left(1^{\lambda_{1}}, 2^{\lambda_{2}}, \ldots, n^{\lambda_{n}}\right)
$$


depending on $n$ external momenta $k_{i}$ and helicities $\lambda_{i}$. The momenta need not be on-shell, but momentum conservation is imposed:

$$
\sum_{\text {gluons }} k_{l}+\sum_{\text {outgoing quarks }} k_{i}-\sum_{\text {incoming quarks }} k_{j}=0 .
$$

By definition, the off-shell amplitudes $O_{n}$ are calculated from all Feynman diagrams contributing to the cyclic-ordered partial amplitude $A_{n}$, by using the off-shell continuation eq. (11) for all external spinors and polarisation vectors, and by using the axial gauge for all internal gluon propagators. Compared to off-shell currents, which are used in recurrence relations of BerendsGiele type, an off-shell amplitude may have more than one leg off-shell. By construction, if all external particles are on-shell, the off-shell amplitude $O_{n}$ coincides with the physical amplitude $A_{n}$. Let $n_{-}$be the number of partons with negative helicity of the off-shell amplitude $O_{n}$. Then we define the degree $d$ of the off-shell amplitude $O_{n}$ as

$$
d=n_{-}-1
$$

\subsection{The pure gluonic case}

We may continue the expressions for the gluon polarisation vectors in eq. (9) to $k^{2} \neq 0$, by using the projection onto $k^{b}$ :

$$
\varepsilon_{\mu}^{+}(k, q)=\frac{\left\langle q-\left|\gamma_{\mu}\right| k^{b}-\right\rangle}{\sqrt{2}\left\langle q-\mid k^{b}+\right\rangle}, \quad \varepsilon_{\mu}^{-}(k, q)=\frac{\left\langle q+\left|\gamma_{\mu}\right| k^{b}+\right\rangle}{\sqrt{2}\left\langle k^{b}+\mid q-\right\rangle} .
$$

The off-shell continued polarisation vectors satisfy:

$$
\varepsilon_{\mu}^{ \pm}(k, q) k^{\mu}=0, \quad \varepsilon_{\mu}^{ \pm}(k, q) q^{\mu}=0, \quad \varepsilon_{\mu}^{+}\left(k_{1}, q\right) \varepsilon^{+\mu}\left(k_{2}, q\right)=0, \quad \varepsilon_{\mu}^{-}\left(k_{1}, q\right) \varepsilon^{-\mu}\left(k_{2}, q\right)=0 .
$$

The polarisation sum is

$$
\sum_{\lambda=+/-} \varepsilon_{\mu}^{\lambda}(k, q) \varepsilon_{v}^{-\lambda}(k, q)=-g_{\mu v}+2 \frac{k_{\mu}^{b} q_{v}+q_{\mu} k_{v}^{b}}{2 k q} .
$$

The gluon propagator in the axial gauge is given by

$$
\frac{i}{k^{2}} d_{\mu v}=\frac{i}{k^{2}}\left(-g_{\mu v}+2 \frac{k_{\mu} q_{v}+q_{\mu} k_{v}}{2 k q}\right) .
$$

If we introduce an unphysical polarisation

$$
\varepsilon_{\mu}^{0}(k, q)=2 \frac{\sqrt{k^{2}}}{2 k q} q_{\mu}
$$

the propagator can be written as

$$
\frac{i}{k^{2}} d_{\mu v}=\frac{i}{k^{2}}\left(\varepsilon_{\mu}^{+} \varepsilon_{v}^{-}+\varepsilon_{\mu}^{-} \varepsilon_{v}^{+}+\varepsilon_{\mu}^{0} \varepsilon_{v}^{0}\right) .
$$



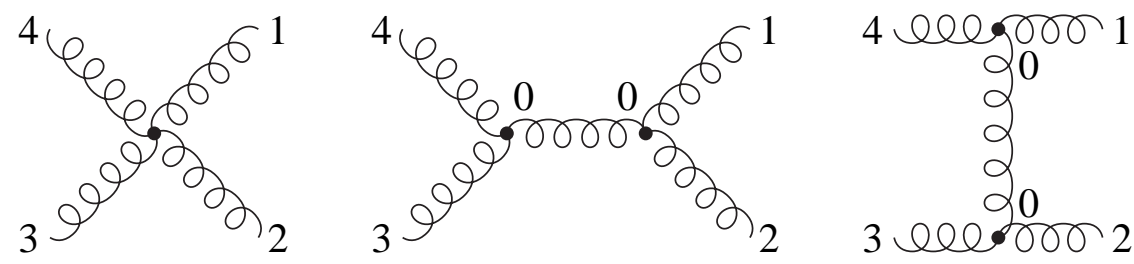

Figure 1: Diagrams contributing to the four-valent gluon vertex. In the last two diagrams only the $\varepsilon^{0}$ polarisation is exchanged.

This has already the structure of a scalar propagator $i / k^{2}$ times a sum over polarisations. The additional polarisation $\varepsilon^{0}$ can be absorbed into a redefinition of the four-gluon vertex. This has already been noted in [12]. We define three- and four-valent primitive vertices as follows: The three-valent vertex is just is just the three-gluon vertex contracted with off-shell polarisation vectors:

$$
V_{3}\left(1^{\lambda_{1}}, 2^{\lambda_{2}}, 3^{\lambda_{3}}\right)=O_{3}\left(1^{\lambda_{1}}, 2^{\lambda_{2}}, 3^{\lambda_{3}}\right) .
$$

From the properties of the polarisation vectors it follows, that $V_{3}\left(1^{+}, 2^{+}, 3^{+}\right)$and $V_{3}\left(1^{-}, 2^{-}, 3^{-}\right)$ vanish. Non-zero values are obtained for

$$
\begin{aligned}
& V_{3}\left(1^{-}, 2^{-}, 3^{+}\right)=i \sqrt{2}\langle 12\rangle \frac{[3 q]^{2}}{[1 q][2 q]}=i \sqrt{2} \frac{\langle 12\rangle^{4}}{\langle 12\rangle\langle 23\rangle\langle 31\rangle} \\
& V_{3}\left(1^{+}, 2^{+}, 3^{-}\right)=i \sqrt{2}[21] \frac{\langle 3 q\rangle^{2}}{\langle 1 q\rangle\langle 2 q\rangle}=i \sqrt{2} \frac{[21]^{4}}{[32][21][13]}
\end{aligned}
$$

and cyclic permutations thereof. Let us now consider the polarisation $\varepsilon_{\mu}^{0}$. The contraction of $\varepsilon_{\mu}^{0}$ with $\varepsilon^{+\mu}, \varepsilon^{-\mu}$ or $\varepsilon^{0 \mu}$ vanishes. Therefore any contraction of $\varepsilon_{\mu}^{0}$ into a four-gluon vertex will give a vanishing contribution. The only non-zero contribution is obtained from a contraction of a single $\varepsilon^{0}$ into a three-gluon vertex. In this case the other helicities are necessarily $\varepsilon^{+}$and $\varepsilon^{-}$. It follows that on both ends of a $\varepsilon_{\mu}^{0} \varepsilon_{v}^{0}$ propagator there is always a three-gluon vertex attached. Therefore we can absorb the $\varepsilon^{0}$-polarisations into a redefinition of the four-gluon vertex. This leads to the definition of the the primitive four-valent vertex, which is obtained from the sum of the standard four-gluon vertex plus contributions resulting from two three-gluon vertices and an $\varepsilon_{\mu}^{0} \varepsilon_{v}^{0}$ propagator. The contributions to the four-valent vertex are shown in fig. 1 . We find that the only non-zero contributions are

$$
\begin{aligned}
V_{4}\left(1^{+}, 2^{+}, 3^{-}, 4^{-}\right)= & i \frac{[1 q][2 q]\langle 3 q\rangle\langle 4 q\rangle}{\langle 1 q\rangle\langle 2 q\rangle[3 q][4 q]}\left(1+\frac{\langle q-|2-3| q-\rangle\langle q-|4-1| q-\rangle}{\langle q-|2+3| q-\rangle\langle q-|4+1| q-\rangle}\right), \\
V_{4}\left(1^{+}, 2^{-}, 3^{+}, 4^{-}\right)= & i \frac{[1 q]\langle 2 q\rangle[3 q]\langle 4 q\rangle}{\langle 1 q\rangle[2 q]\langle 3 q\rangle[4 q]}\left(\frac{\langle q-|1-2| q-\rangle\langle q-|3-4| q-\rangle}{\langle q-|1+2| q-\rangle\langle q-|3+4| q-\rangle}\right. \\
& \left.+\frac{\langle q-|2-3| q-\rangle\langle q-|4-1| q-\rangle}{\langle q-|2+3| q-\rangle\langle q-|4+1| q-\rangle}-2\right),
\end{aligned}
$$


and permutations thereof. We could in principle eliminate the explicit dependence on the reference vector $q$, by noting that

$$
k_{1}^{b}+k_{2}^{b}+k_{3}^{b}+k_{4}^{b}=-\left(\frac{k_{1}^{2}}{2 k_{1} q}+\frac{k_{2}^{2}}{2 k_{2} q}+\frac{k_{3}^{2}}{2 k_{3} q}+\frac{k_{4}^{2}}{2 k_{4} q}\right) q .
$$

For example, the prefactor becomes in this case

$$
\frac{[1 q]\langle 2 q\rangle[3 q]\langle 4 q\rangle}{\langle 1 q\rangle[2 q]\langle 3 q\rangle[4 q]}=\frac{\langle 1+|2+3| 4+\rangle\langle 2+|4+1| 3+\rangle}{\langle 1-|2+3| 4-\rangle\langle 2-|4+1| 3-\rangle} .
$$

Let $n_{-}$be the number of particles with negative helicity of a vertex. As for off-shell amplitudes we define the degree $d$ of a vertex as

$$
d=n_{-}-1
$$

We observe that for each vertex we have at least one parton of negative helicity, but never more than two parton with negative helicity. Therefore all vertices are of degree zero or one.

\subsection{Quarks}

The off-shell continuation to $p^{2} \neq m^{2}$ for spinors is straightforward:

$$
\begin{array}{ll}
u(-)=\left|p^{b}+\right\rangle+\frac{m}{\left\langle p^{b}+\mid q-\right\rangle}|q-\rangle, & \bar{u}(+)=\left\langle p^{b}+\right|+\frac{m}{\left\langle q-\mid p^{b}+\right\rangle}\langle q-|, \\
u(+)=\left|p^{b}-\right\rangle+\frac{m}{\left\langle p^{b}-\mid q+\right\rangle}|q+\rangle, & \bar{u}(-)=\left\langle p^{b}-\right|+\frac{m}{\left\langle q+\mid p^{b}-\right\rangle}\langle q+| .
\end{array}
$$

These spinors have a smooth massless limit and the off-shell continuation of a massless quark is simply

$$
\begin{array}{ll}
u(-)=\left|p^{b}+\right\rangle, & \bar{u}(+)=\left\langle p^{b}+\right| \\
u(+)=\left|p^{b}-\right\rangle, & \bar{u}(-)=\left\langle p^{b}-\right| .
\end{array}
$$

It is therefore sufficient to discuss the generic case of massive quarks. Massless quarks are obtained by the $m \rightarrow 0$ limit. The polarisation sum of the spinors in eq. (28) reads

$$
\sum_{\lambda=+/-} u(-\lambda) \bar{u}(\lambda)=\not+m-\frac{p^{2}-m^{2}}{2 p q} \not 1 .
$$

Therefore we can rewrite the quark propagator as

$$
i \frac{p+m}{p^{2}-m^{2}}=\frac{i}{p^{2}-m^{2}}\left(\sum_{\lambda=+/-} u(-\lambda) \bar{u}(\lambda)+\frac{p^{2}-m^{2}}{2 p q} \phi\right) .
$$



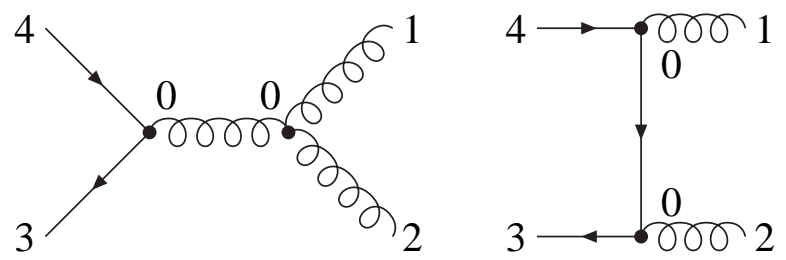

Figure 2: Diagrams contributing to the four-valent quark-gluon vertex. For the internal propagator only " 0 "-polarisations are exchanged.

We note the similarity with eq. (21). As the gluon propagator, the quark propagator can be written as a polarisation sum of the off-shell continued polarisation vectors and a piece proportional to q. We say that this piece describes the exchange of an unphysical "0"-polarisation. Again, we can re-absorb the piece proportional to $\not$ into a four-valent vertex. To do so, we have to take into account that also a gluon with an $\varepsilon^{0}$-polarisation can couple to a quark-gluon vertex. It is easily verified, that for a quark-gluon vertex we can have no more than one "0"-polarisation coupling to this vertex. (If we contract two or more "0"-polarisations into the vertex, we obtain zero.) This ensures that on both ends of propagators carrying the " 0 "-polarisation we have vertices, which contain only the physical "+/-" polarisations. Therefore the effect of the exchange of " 0 "polarisations can be absorbed into the definition of a four-valent vertex. Let us now discuss the additional vertices due to the inclusion of quarks: First, there is a three-valent vertex, obtained from the quark-gluon vertex contracted with the off-shell polarisation vectors.

$$
V_{3}\left(1_{q}^{\lambda_{1}}, 2_{\bar{q}}^{\lambda_{2}}, 3^{\lambda_{3}}\right)=O_{3}\left(1_{q}^{\lambda_{1}}, 2_{\bar{q}}^{\lambda_{2}}, 3^{\lambda_{3}}\right) .
$$

Non-zero contributions are obtained for massless or massive quarks for the helicity configurations

$$
V_{3}\left(1_{q}^{+}, 2_{\bar{q}}^{-}, 3^{+}\right), \quad V_{3}\left(1_{q}^{+}, 2_{\bar{q}}^{-}, 3^{-}\right), \quad V_{3}\left(1_{q}^{-}, 2_{\bar{q}}^{+}, 3^{+}\right), \quad V_{3}\left(1_{q}^{-}, 2_{\bar{q}}^{+}, 3^{-}\right) .
$$

For massive quarks there are two additional helicity configurations

$$
V_{3}\left(1_{q}^{+}, 2_{\bar{q}}^{+}, 3^{-}\right), \quad V_{3}\left(1_{q}^{-}, 2_{\bar{q}}^{-}, 3^{+}\right),
$$

which induce a spin-flip along the quark line. These vertices are proportional to the mass of the quark and vanish in the massless limit.

The absorbtion of the unphysical "0"-polarisations leads to two new four-valent vertices. First, there is a vertex

$$
V_{4}\left(1^{\lambda_{1}}, 2^{\lambda_{2}}, 3_{q}^{\lambda_{3}}, 4_{\bar{q}}^{\lambda_{4}}\right)
$$

involving two quarks and two gluons. The Feynman diagrams contributing to this vertex are shown in fig. 2. Non-zero contributions are obtained for the helicity configurations

$$
V_{4}\left(1^{+}, 2^{-}, 3_{q}^{+}, 4_{\bar{q}}^{-}\right), \quad V_{4}\left(1^{-}, 2^{+}, 3_{q}^{+}, 4_{\bar{q}}^{-}\right), \quad V_{4}\left(1^{+}, 2^{-}, 3_{q}^{-}, 4_{\bar{q}}^{+}\right), \quad V_{4}\left(1^{-}, 2^{+}, 3_{q}^{-}, 4_{\bar{q}}^{+}\right) \text {. }
$$




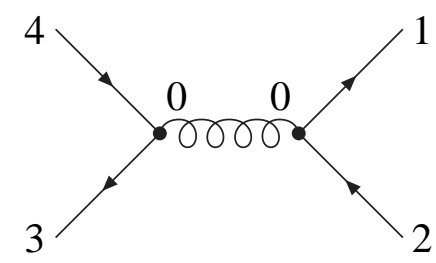

Figure 3: Diagrams contributing to the four-valent quark-quark vertex. For the internal propagator only "0"-polarisations are exchanged.

Secondly, there is a vertex

$$
V_{4}\left(1_{q}^{\lambda_{1}}, 2_{\bar{q}}^{\lambda_{2}}, 3_{q}^{\lambda_{3}}, 4_{\bar{q}}^{\lambda_{4}}\right)
$$

involving two pairs of quarks. The Feynman diagram contributing to this vertex is shown in fig. 3. Non-zero contributions are obtained for the helicity configurations

$$
V_{4}\left(1_{q}^{+}, 2_{\bar{q}}^{-}, 3_{q}^{+}, 4_{\bar{q}}^{-}\right), \quad V_{4}\left(1_{q}^{+}, 2_{\bar{q}}^{-}, 3_{q}^{-}, 4_{\bar{q}}^{+}\right) .
$$

We have summarised the formulae for all non-vanishing vertices in appendix B. As in the pure gluon case, all vertices are of degree zero or one.

\section{Discussion}

In the previous section we have shown, that all QCD amplitudes can be calculated from a set of three- or four-valent vertices and scalar propagators. We have defined the degree of a vertex as the number of negative helicities minus one. As each vertex has either one or two negative helicities, we deal only with vertices of degree zero or one. Further, one observes that all vertices of degree zero are three-valent, whereas vertices of degree one can either be three-valent or fourvalent. We further defined the degree of an off-shell amplitude as the number of the external particles with negative helicity minus one. As an off-shell amplitude is a sum of diagrams, each diagram inherits the degree of the off-shell amplitude. We would like to show that the degree of a diagram is the sum of the degrees of its vertices. To this aim let $v_{3}^{+}$be the number of three-valent vertices of degree zero in the diagram. Similar, let $v_{3}^{-}$be the number of three-valent vertices of degree one and let $v_{4}^{-}$be the number of four-valent vertices of degree one. (Note that there are no four-valent vertices of degree zero.) Since we are considering Born diagrams, the number of internal propagators $n_{\text {prop }}$ is just the number of vertices minus one:

$$
n_{\text {prop }}=v_{3}^{+}+v_{3}^{-}+v_{4}^{-}-1 \text {. }
$$

In total we have

$$
v_{3}^{+}+2 v_{3}^{-}+2 v_{4}^{-}
$$


negative helicity labels in the diagram. As a propagator connects "+"-helicities with "-"-helicities, $n_{\text {prop }}$ negative helicity labels are attached to internal propagators. Therefore the number $n_{-}$of external particles with negative helicity is given by

$$
n_{-}=v_{3}^{-}+v_{4}^{-}+1
$$

Therefore the degree $d$ of the diagram is given by

$$
d=n_{-}-1=v_{3}^{-}+v_{4}^{-},
$$

which shows that the degree $d$ of a diagram is the sum of the degrees of its vertices.

Let us consider the pure gluon off-shell amplitude $O_{n}\left(1^{+}, 2^{+}, \ldots, n^{+}\right)$, where all gluons have positive helicity. This amplitude would have degree $(-1)$. Since there are no vertices of negative degree, this off-shell amplitude must vanish. This actually proves that the first Parke-Taylor formula still holds if all particles are continued off-shell.

Of particular importance are off-shell amplitude of degree zero and one. From the argument above it follows that off-shell amplitudes of degree zero must contain only vertices of degree zero. In the pure gluonic case, there is only one degree zero vertex. As a consequence, the pure gluonic off-shell amplitude $O_{n}\left(1^{+}, 2^{+}, \ldots,(n-1)^{+}, n^{-}\right)$of degree zero fulfills a rather simple recurrence relation:

$$
\begin{aligned}
& O_{n}\left(1^{+}, \ldots,(n-1)^{+}, n^{-}\right)=\sum_{j=2}^{n-1} V_{3}\left(\left(-k_{1, j}\right)^{+},\left(-k_{j, n}\right)^{+}, n^{-}\right) \\
& \quad \times \frac{i}{k_{1, j}^{2}} O_{j}\left(1^{+}, \ldots,(j-1)^{+}, k_{1, j}^{-}\right) \frac{i}{k_{j, n}^{2}} O_{n-j+1}\left(j^{+}, \ldots,(n-1)^{+}, k_{j, n}^{-}\right),
\end{aligned}
$$

where

$$
k_{i, j}=\sum_{l=i}^{j-1} k_{l},
$$

and where we define the two-point amplitude to be the inverse propagator:

$$
O_{2}\left((j-1)^{+}, k_{(j-1), j}^{-}\right)=-i k_{(j-1), j}^{2} .
$$

Off-shell amplitudes of degree one contain exactly one vertex of degree one. All other vertices are of degree zero. Since by definition an off-shell amplitude is just a sum of diagrams build out of the basic vertices in all possible ways compatible with the cyclic ordering, an off-shell amplitude of degree one consists therefore of diagrams containing a vertex of degree one dressed up in all possible ways compatible with the cyclic ordering with vertices of degree zero.

Let us now consider an off-shell amplitude with $n_{-}$external particles of negative helicity. This amplitude is of degree $d=n_{-}-1$ and each diagram has exactly $\left(n_{-}-1\right)$ vertices of degree one. The off-shell amplitude is just the sum of all diagrams, obtained from combining in all possible ways compatible with the cyclic ordering $\left(n_{-}-1\right)$ vertices of degree one with the remaining vertices of degree zero. 
We would like to comment on amplitudes with massive quarks. Compared to the massless case, two new features make their appearance: First there are helicity configurations, which vanish in the simultaneous massless and on-shell limit, but remain non-zero in the on-shell limit for non-zero masses. Examples of this kind are amplitudes of the form

$$
A_{n}\left(1_{Q}^{+}, 2_{\bar{Q}}^{-}, 3^{+}, \ldots, n^{+}\right)
$$

Secondly, there are the helicity flip vertices

$$
V_{3}\left(1_{q}^{+}, 2_{\bar{q}}^{+}, 3^{-}\right), \quad V_{3}\left(1_{q}^{-}, 2_{\bar{q}}^{-}, 3^{+}\right),
$$

which are proportional to the mass and vanish therefore in the massless limit. We note that $V_{3}\left(1_{q}^{+}, 2_{\bar{q}}^{+}, 3^{-}\right)$is a degree zero vertex, whereas $V_{3}\left(1_{q}^{-}, 2_{\bar{q}}^{-}, 3^{+}\right)$has degree one. Let us consider an amplitude with one massive quark pair. We first consider the helicity configuration $Q^{+} \bar{Q}^{-}$. Along the massive quark line we must have an equal number of helicity flips induced by $V_{3}\left(1_{q}^{+}, 2_{\bar{q}}^{+}, 3^{-}\right)$ and $V_{3}\left(1_{q}^{-}, 2_{\bar{q}}^{-}, 3^{+}\right)$. Since the latter vertex is of degree one, the total number of helicty flips $f$ is bounded by the degree $d$ of the amplitude:

$$
f \leq 2 d
$$

As an example it follows immediately from this bound that amplitudes of the form (46) cannot contain a helicity flip. A similar argument applies to the helicity configuration $Q^{+} \bar{Q}^{+}$. Here we have along the fermion line at least one helicity flip of degree zero together with at most $d$ additional pairs of degree one and zero helicity flips. So the total number is bounded by

$$
f \leq 2 d+1
$$

For the helicity configuration $Q^{-} \bar{Q}^{-}$there is at least one flip of degree one, leaving at most $2(d-1)$ additional helicity flips so the total number is bounded by

$$
f \leq 2 d-1
$$

\section{Conclusions}

In this paper we considered Born amplitudes in QCD. Decomposing the full amplitude into gauge-invariant partial amplitudes, we showed that the partial amplitudes can be calculated from simple rules, involving only scalar propagators and a set of complex valued three- and four-valent vertices. As no contraction of Lorentz- or spinor-indices is present any more, our method is well suited for a fast implementation on a computer. Our approach is not restricted to gluons only, but treated gluons, massless quarks and massive quarks on almost equal footing. It is possible to assign to each vertex a degree, given by the number of negative helicities minus one. It is a striking feature, that only vertices of degree zero and one occur. 


\section{Acknowledgements}

The work of C.S. has been supported by the Deutsche Forschungsgemeinschaft through the Graduiertenkolleg 'Eichtheorien' at Mainz University.

\section{A Spinors}

For the metric we use

$$
g_{\mu \nu}=\operatorname{diag}(+1,-1,-1,-1) .
$$

We define the light-cone coordinates as

$$
p_{+}=p_{0}+p_{3}, \quad p_{-}=p_{0}-p_{3}, \quad p_{\perp}=p_{1}+i p_{2}, \quad p_{\perp *}=p_{1}-i p_{2}
$$

In terms of the light-cone components of a null-vector, the corresponding massless spinors $\langle p \pm|$ and $|p \pm\rangle$ can be chosen as

$$
\begin{gathered}
|p+\rangle=\frac{e^{-i \frac{\phi}{2}}}{\sqrt{\left|p_{+}\right|}}\left(\begin{array}{c}
-p_{\perp^{*}} \\
p_{+}
\end{array}\right), \quad|p-\rangle=\frac{e^{-i \frac{\phi}{2}}}{\sqrt{\left|p_{+}\right|}}\left(\begin{array}{c}
p_{+} \\
p_{\perp}
\end{array}\right), \\
\langle p+|=\frac{e^{-i \frac{\phi}{2}}}{\sqrt{\left|p_{+}\right|}}\left(-p_{\perp}, p_{+}\right), \quad\langle p-|=\frac{e^{-i \frac{\phi}{2}}}{\sqrt{\left|p_{+}\right|}}\left(p_{+}, p_{\perp *}\right),
\end{gathered}
$$

where the phase $\phi$ is given by

$$
p_{+}=\left|p_{+}\right| e^{i \phi_{+}} .
$$

With these phase conventions we have the following relations between a spinor corresponding to a vector $p$ and a spinor corresponding to a vector $(-p)$ :

$$
\begin{aligned}
& |(-p) \pm\rangle=i|p \pm\rangle, \\
& \langle(-p) \pm|=i\langle p \pm| .
\end{aligned}
$$

Consequently, we have for the polarisation vectors of the gluon:

$$
\varepsilon_{\mu}^{ \pm}(-k, q)=\varepsilon_{\mu}^{ \pm}(k, q)
$$

For the quarks we take the momentum to flow always along the fermion line, i.e. we only consider in- and outgoing quarks with spinors $u$ and $\bar{u}$. In- and outgoing anti-quarks with spinors $v$ and $\bar{v}$ are related to the spinors $u$ and $\bar{u}$ by taking the momenta $p$ to $-p$ and a flip in helicity. For massive quarks we have the spinors

$$
\begin{aligned}
& u(+)=\frac{1}{\left\langle p^{b}-\mid q+\right\rangle}(\not p+m)|q+\rangle=\left|p^{b}-\right\rangle+\frac{m}{\left\langle p^{b}-\mid q+\right\rangle}|q+\rangle, \\
& u(-)=\frac{1}{\left\langle p^{b}+\mid q-\right\rangle}(\not p+m)|q-\rangle=\left|p^{b}+\right\rangle+\frac{m}{\left\langle p^{b}+\mid q-\right\rangle}|q-\rangle .
\end{aligned}
$$


For the conjugate spinors we have

$$
\begin{aligned}
& \bar{u}(+)=\frac{1}{\left\langle q-\mid p^{b}+\right\rangle}\langle q-|(p \mid+m)=\left\langle p^{b}+\right|+\frac{m}{\left\langle q-\mid p^{b}+\right\rangle}\langle q-|, \\
& \bar{u}(-)=\frac{1}{\left\langle q+\mid p^{b}-\right\rangle}\langle q+|(p \mid+m)=\left\langle p^{b}-\right|+\frac{m}{q+\left|p^{b}-\right\rangle}\langle q+| .
\end{aligned}
$$

These spinors satisfy the Dirac equations

$$
\left(\not p^{\prime}-m\right) u(\lambda)=0, \quad \bar{u}(\lambda)(\not p-m)=0,
$$

the orthogonality relations

$$
\bar{u}(\bar{\lambda}) u(-\lambda)=2 m \delta_{\bar{\lambda} \lambda}
$$

and the completeness relation

$$
\sum_{\lambda=+/-} u(-\lambda) \bar{u}(\lambda)=\not l+m
$$

Note that these spinors have a smooth massless limit. For $m=0$ we obtain

$$
u( \pm)=|p \mp\rangle, \quad \bar{u}( \pm)=\langle p \pm| .
$$

\section{B Summary of the diagrammatic rules}

In this section we summarise the diagrammatic rules. Propagators are always scalars, e.g.

$$
\frac{i}{p^{2}}
$$

for gluons and massless quarks, and

$$
\frac{i}{p^{2}-m^{2}}
$$

for massive quarks. They connect "+"-labels with "-"-labels. The expressions for the vertices involve spinor products. We recall our notation:

$$
\begin{aligned}
& \langle i j\rangle=\left\langle k_{i}^{b}-\mid k_{j}^{b}+\right\rangle, \quad[i j]=\left\langle k_{i}^{b}+\mid k_{j}^{b}-\right\rangle, \\
& \langle i-|j \pm k| l-\rangle=\left\langle k_{i}^{b}-\left|k_{j}^{b} \pm k_{k}^{b}\right| k_{l}^{b}-\right\rangle,
\end{aligned}
$$

where $k^{b}$ is the projected light-like four-vector associated to $k . k^{b}$ is obtained from $k$ and the reference momentum $q$ as follows:

$$
k^{b}=k-\frac{k^{2}}{2 k q} q \text {. }
$$


Our conventions for the momenta are such that we always take all gluon momenta outgoing and the momenta of quarks to flow in the direction of the fermion lines. The vertices involving only gluons are:

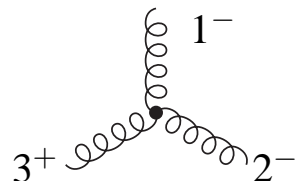

$$
=i \sqrt{2}\langle 12\rangle \frac{[3 q]^{2}}{[1 q][2 q]}=i \sqrt{2} \frac{\langle 12\rangle^{4}}{\langle 12\rangle\langle 23\rangle\langle 31\rangle}
$$

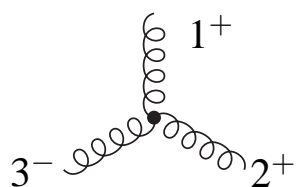

$$
=i \sqrt{2}[21] \frac{\langle 3 q\rangle^{2}}{\langle 1 q\rangle\langle 2 q\rangle}=i \sqrt{2} \frac{[21]^{4}}{[32][21][13]}
$$

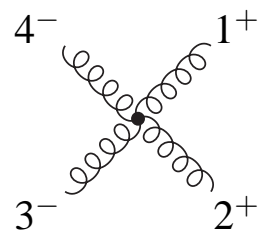

$$
=i \frac{[1 q][2 q]\langle 3 q\rangle\langle 4 q\rangle}{\langle 1 q\rangle\langle 2 q\rangle[3 q][4 q]}\left(1+\frac{\langle q-|2-3| q-\rangle\langle q-|4-1| q-\rangle}{\langle q-|2+3| q-\rangle\langle q-|4+1| q-\rangle}\right),
$$

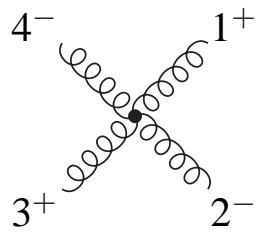

$$
\begin{aligned}
= & i \frac{[1 q]\langle 2 q\rangle[3 q]\langle 4 q\rangle}{\langle 1 q\rangle[2 q]\langle 3 q\rangle[4 q]}\left(\frac{\langle q-|1-2| q-\rangle\langle q-|3-4| q-\rangle}{\langle q-|1+2| q-\rangle\langle q-|3+4| q-\rangle}\right. \\
& \left.+\frac{\langle q-|2-3| q-\rangle\langle q-|4-1| q-\rangle}{\langle q-|2+3| q-\rangle\langle q-|4+1| q-\rangle}-2\right) .
\end{aligned}
$$

Three-valent vertices involving a pair of quarks are:

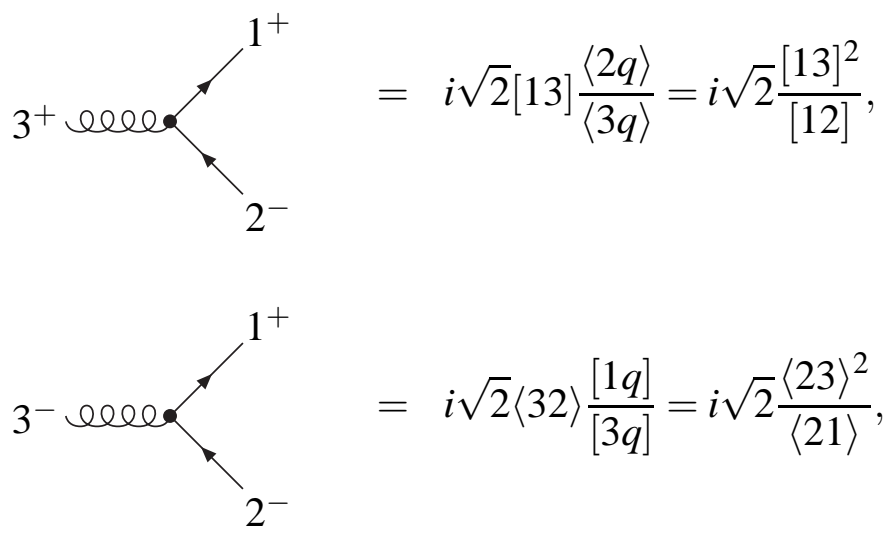




$$
\begin{aligned}
& 3_{2^{+}}^{1^{-}}=i \sqrt{2}[23] \frac{\langle 1 q\rangle}{\langle 3 q\rangle}=i \sqrt{2} \frac{[23]^{2}}{[12]} \\
& 3_{2^{+}}^{1^{-}}=i \sqrt{2}\langle 31\rangle \frac{[2 q]}{[3 q]}=i \sqrt{2} \frac{\langle 31\rangle^{2}}{\langle 21\rangle} \\
& 3_{2^{+}}^{1^{+}}=-i \sqrt{2} m \frac{\langle 3 q\rangle^{2}}{\langle 1 q\rangle\langle 2 q\rangle}=i \sqrt{2} m \frac{[12]^{2}}{[23][31]} \\
& 3_{2^{-}}^{1^{-}}=i \sqrt{2} m \frac{[3 q]^{2}}{[1 q][2 q]}=-i \sqrt{2} m \frac{\langle 12\rangle^{2}}{\langle 23\rangle\langle 31\rangle}
\end{aligned}
$$

Note that the last two vertices involve a helicity flip along the fermion line and vanish in the massless limit. The four-valent vertices with a pair of quarks and two gluons are:

$$
\begin{aligned}
& \int_{2}^{6^{6}} 2_{2}^{1^{+}} \\
& =2 i \frac{[1 q]\langle 2 q\rangle}{\langle 1 q\rangle[2 q]} \frac{[3 q]\langle 4 q\rangle}{\langle q-|3-4| q-\rangle}\left(\frac{\langle q-|1-2| q-\rangle}{\langle q-|1+2| q-\rangle}+\frac{\langle q-|3-4| q-\rangle}{\langle q-|2+3| q-\rangle}\right), \\
& \int_{3^{+}}^{6^{6}} \\
& =2 i \frac{\langle 1 q\rangle[2 q]}{[1 q]\langle 2 q\rangle} \frac{[3 q]\langle 4 q\rangle}{\langle q-|3-4| q-\rangle} \frac{\langle q-|1-2| q-\rangle}{\langle q-|1+2| q-\rangle}
\end{aligned}
$$




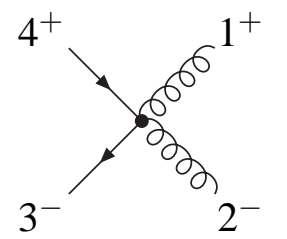

$$
=2 i \frac{[1 q]\langle 2 q\rangle}{\langle 1 q\rangle[2 q]} \frac{\langle 3 q\rangle[4 q]}{\langle q-|3-4| q-\rangle} \frac{\langle q-|1-2| q-\rangle}{\langle q-|1+2| q-\rangle}
$$

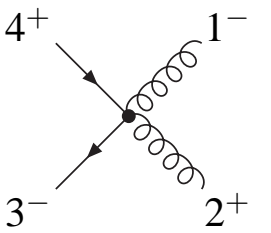

$$
=2 i \frac{\langle 1 q\rangle[2 q]}{[1 q]\langle 2 q\rangle} \frac{\langle 3 q\rangle[4 q]}{\langle q-|3-4| q-\rangle}\left(\frac{\langle q-|1-2| q-\rangle}{\langle q-|1+2| q-\rangle}+\frac{\langle q-|3-4| q-\rangle}{\langle q-|2+3| q-\rangle}\right) .
$$

Finally, we have the four-valent vertices with two pairs of quarks:

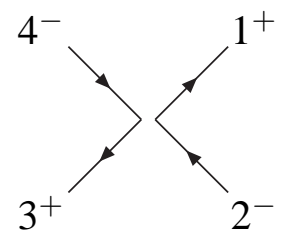

$$
=4 i \frac{[1 q]\langle 2 q\rangle[3 q]\langle 4 q\rangle}{\langle q-|1-2| q-\rangle\langle q-|3-4| q-\rangle},
$$

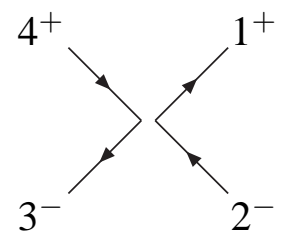

$$
=4 i \frac{[1 q]\langle 2 q\rangle\langle 3 q\rangle[4 q]}{\langle q-|1-2| q-\rangle\langle q-|3-4| q-\rangle} .
$$

\section{References}

[1] P. Cvitanovic, P. G. Lauwers, and P. N. Scharbach, Nucl. Phys. B186, 165 (1981).

[2] F. A. Berends and W. Giele, Nucl. Phys. B294, 700 (1987).

[3] M. L. Mangano, S. J. Parke, and Z. Xu, Nucl. Phys. B298, 653 (1988).

[4] D. Kosower, B.-H. Lee, and V. P. Nair, Phys. Lett. B201, 85 (1988).

[5] Z. Bern and D. A. Kosower, Nucl. Phys. B362, 389 (1991).

[6] F. A. Berends, R. Kleiss, P. De Causmaecker, R. Gastmans, and T. T. Wu, Phys. Lett. B103, 124 (1981).

[7] P. De Causmaecker, R. Gastmans, W. Troost, and T. T. Wu, Nucl. Phys. B206, 53 (1982). 
[8] J. F. Gunion and Z. Kunszt, Phys. Lett. B161, 333 (1985).

[9] Z. Xu, D.-H. Zhang, and L. Chang, Nucl. Phys. B291, 392 (1987).

[10] R. Gastmans and T. T. Wu, Oxford, UK: Clarendon (1990) 648 p. (International series of monographs on physics, 80 ).

[11] F. A. Berends and W. T. Giele, Nucl. Phys. B306, 759 (1988).

[12] D. A. Kosower, Nucl. Phys. B335, 23 (1990).

[13] S. J. Parke and T. R. Taylor, Phys. Rev. Lett. 56, 2459 (1986).

[14] F. Cachazo, P. Svrcek, and E. Witten, JHEP 09, 006 (2004), hep-th/0403047.

[15] E. Witten, Commun. Math. Phys. 252, 189 (2004), hep-th/0312171.

[16] R. Roiban, M. Spradlin, and A. Volovich, JHEP 04, 012 (2004), hep-th/0402016.

[17] R. Roiban and A. Volovich, Phys. Rev. Lett. 93, 131602 (2004), hep-th/0402121.

[18] R. Roiban, M. Spradlin, and A. Volovich, Phys. Rev. D70, 026009 (2004), hep-th/0403190.

[19] S. Gukov, L. Motl, and A. Neitzke, (2004), hep-th/0404085.

[20] D. A. Kosower, Phys. Rev. D71, 045007 (2005), hep-th/0406175.

[21] C.-J. Zhu, JHEP 04, 032 (2004), hep-th/0403115.

[22] F. Cachazo, P. Svrcek, and E. Witten, JHEP 10, 074 (2004), hep-th/0406177.

[23] F. Cachazo, P. Svrcek, and E. Witten, JHEP 10, 077 (2004), hep-th/0409245.

[24] F. Cachazo, (2004), hep-th/0410077.

[25] R. Britto, F. Cachazo, and B. Feng, Phys. Rev. D71, 025012 (2005), hep-th/0410179.

[26] R. Britto, F. Cachazo, and B. Feng, (2004), hep-th/0411107.

[27] R. Britto, F. Cachazo, and B. Feng, (2004), hep-th/0412103.

[28] I. Bena, Z. Bern, D. A. Kosower, and R. Roiban, (2004), hep-th/0410054.

[29] Z. Bern, V. Del Duca, L. J. Dixon, and D. A. Kosower, Phys. Rev. D71, 045006 (2005), hep-th/0410224.

[30] S. J. Bidder, N. E. J. Bjerrum-Bohr, L. J. Dixon, and D. C. Dunbar, Phys. Lett. B606, 189 (2005), hep-th/0410296. 
[31] S. J. Bidder, N. E. J. Bjerrum-Bohr, D. C. Dunbar, and W. B. Perkins, (2004), hepth/0412023.

[32] Z. Bern, L. J. Dixon, and D. A. Kosower, (2004), hep-th/0412210.

[33] Z. Bern, L. J. Dixon, and D. A. Kosower, (2005), hep-th/0501240.

[34] S. J. Bidder, N. E. J. Bjerrum-Bohr, D. C. Dunbar, and W. B. Perkins, (2005), hepth/0502028.

[35] A. Brandhuber, B. Spence, and G. Travaglini, Nucl. Phys. B706, 150 (2005), hepth/0407214.

[36] J. Bedford, A. Brandhuber, B. Spence, and G. Travaglini, Nucl. Phys. B706, 100 (2005), hep-th/0410280.

[37] J. Bedford, A. Brandhuber, B. Spence, and G. Travaglini, (2004), hep-th/0412108.

[38] C. Quigley and M. Rozali, JHEP 01, 053 (2005), hep-th/0410278.

[39] R. Roiban, M. Spradlin, and A. Volovich, (2004), hep-th/0412265.

[40] M.-x. Luo and C.-k. Wen, JHEP 11, 004 (2004), hep-th/0410045.

[41] M.-x. Luo and C.-k. Wen, (2004), hep-th/0410118.

[42] G. Chalmers and W. Siegel, Phys. Rev. D59, 045012 (1999), hep-ph/9708251.

[43] G. Chalmers and W. Siegel, Phys. Rev. D59, 045013 (1999), hep-ph/9801220.

[44] G. Chalmers and W. Siegel, Phys. Rev. D63, 125027 (2001), hep-th/0101025.

[45] W. Siegel, (1999), hep-th/9912205.

[46] I. Bena, Z. Bern, and D. A. Kosower, (2004), hep-th/0406133.

[47] R. Britto, F. Cachazo, and B. Feng, (2004), hep-th/0412308.

[48] R. Britto, F. Cachazo, B. Feng, and E. Witten, (2005), hep-th/0501052.

[49] S. J. Parke and T. R. Taylor, Phys. Lett. B157, 81 (1985).

[50] M. T. Grisaru and H. N. Pendleton, Nucl. Phys. B124, 81 (1977).

[51] G. Georgiou and V. V. Khoze, JHEP 05, 070 (2004), hep-th/0404072.

[52] G. Georgiou, E. W. N. Glover, and V. V. Khoze, JHEP 07, 048 (2004), hep-th/0407027.

[53] V. V. Khoze, (2004), hep-th/0408233. 
[54] J.-B. Wu and C.-J. Zhu, JHEP 07, 032 (2004), hep-th/0406085.

[55] J.-B. Wu and C.-J. Zhu, JHEP 09, 063 (2004), hep-th/0406146.

[56] X. Su and J.-B. Wu, (2004), hep-th/0409228.

[57] M.-x. Luo and C.-k. Wen, (2005), hep-th/0501121.

[58] M.-x. Luo and C.-k. Wen, (2005), hep-th/0502009.

[59] Z. Bern, D. Forde, D. A. Kosower, and P. Mastrolia, (2004), hep-ph/0412167.

[60] L. J. Dixon, E. W. N. Glover, and V. V. Khoze, JHEP 12, 015 (2004), hep-th/0411092.

[61] S. D. Badger, E. W. N. Glover, and V. V. Khoze, (2004), hep-th/0412275.

[62] R. Kleiss and W. J. Stirling, Nucl. Phys. B262, 235 (1985).

[63] F. A. Berends, P. H. Daverveldt, and R. Kleiss, Nucl. Phys. B253, 441 (1985).

[64] A. Ballestrero and E. Maina, Phys. Lett. B350, 225 (1995), hep-ph/9403244.

[65] S. Dittmaier, Phys. Rev. D59, 016007 (1999), hep-ph/9805445.

[66] J. van der Heide, E. Laenen, L. Phaf, and S. Weinzierl, Phys. Rev. D62, 074025 (2000), hep-ph/0003318. 